\title{
Phytochemistry and Anti Tyrosinase Activity of Extracts from Leaves of Gynotroches Axillaris (Rhizophoraceae)
}

\author{
Salam Ahmed Abed ${ }^{1, *}$, Hasnah Mohd Sirat ${ }^{1, *}$ and Muhammad Taher ${ }^{2}$ \\ ${ }^{1}$ Department of Chemistry, Faculty of Science, Universiti Teknologi Malaysia, 81310 Skudai, Johor; ${ }^{2}$ Department of \\ Pharmaceutical Technology, Faculty of Pharmacy, Universiti Islam Antarabangsa Malaysia, Kuantan, Pahang; \\ E-mails: salamezee@yahoo.com, hasnah@kimia.fs.utm.my
}

Gynotroches axillaris is the only species of Gynotroches from Rhizophoraceae family. Sequential extraction of powdered leaves of Gynotroches axillaris using petroleum ether, chloroform and methanol afforded three extracts with percentage yield $4.6 \%, 1.6 \%$ and $15 \%$ respectively. Fractionation and purification of petroleum ether extract yielded triterpene ester, $\beta$-amyrin palmitate as the major compound, and two fatty acids, palmitic acid and myristic acid. The methanol extract yielded two flavonoids assigned as quercetin-3-O-L-rhamnoside and catechin. The structures were determined by MS, IR and NMR (1D and 2D). The tyrosinase inhibition assay of petroleum ether, chloroform, methanol extracts and the pure compounds were screened against tyrosinase enzyme. The inhibition percentage (I\%) of methanol extract against tyrosinase enzyme was stronger than the other extracts with I\% values $68.4 \%$. Quercetin-3-O-L-rhamnoside (59\%) was found to be the highest in the tyrosinase inhibition activity compared to standard Kojic acid (83\%), whereas catechin and $\beta$-amyrin palmitate were inactive.

Keywords: Rhizophoraceae, Gynotroches axillaris, Flavonoid, Triterpene ester; Anti Tyrosinase. 